\title{
Combining Graph and Machine Learning Methods to Analyze Differences in Functional Connectivity Across Sex
}

\author{
Casanova R. ${ }^{*}, 1$, Whitlow C.T. ${ }^{2}$, Wagner B. ${ }^{2}$, Espeland M.A. ${ }^{1}$ and Maldjian J.A. ${ }^{2}$ \\ ${ }^{I}$ Department of Biostatistical Sciences ${ }^{2}$ Department of Radiology, Wake Forest University School of Medicine, Winston- \\ Salem, NC, USA
}

\begin{abstract}
In this work we combine machine learning methods and graph theoretical analysis to investigate genderassociated differences in resting state brain network connectivity. The set of all correlations computed from the fMRI resting state data is used as input features for classification. Two ensemble learning methods are used to perform the detection of the set of discriminative edges between groups (males vs. females) of brain networks: 1) Random Forest and 2) an ensemble method based on least angle shrinkage and selection operator (lasso) regressors. Permutation testing is used not only to assess significance of classification accuracy but also to evaluate significance of feature selection. Finally, these methods are applied to data downloaded from the Connectome Project website. Our results suggest that gender differences in brain function may be related to sexually dimorphic regional connectivity between specific critical nodes via gender-discriminative edges.
\end{abstract}

Keywords: Resting state fMRI, Machine learning, Graph theory, Regularization, GLMNET, Random forest.

\section{INTRODUCTION}

The analysis of fMRI resting state (R-fMRI) data has recently emerged as a powerful tool for neuroimaging research. The fact that brain R-fMRI reveals low-frequency fluctuations $(<0.1 \mathrm{~Hz})$ that are temporally correlated across functionally related areas [1-4] implies that it can be used to interrogate multiple brain networks with no prior hypothesis. Graph theory is one of the main approaches employed to evaluate R-fMRI brain network connectivity [5, 6]. Following seminal work describing small world [7] and scale-free networks [8] in the late 1990s, an increasing amount of efforts has been dedicated by the neuroimaging community to characterize the topology of functional [9-13] and anatomical [14-16] brain networks.

A common procedure to generate R-fMRI brain networks consists of the following steps: 1) normalization; 2) motion correction; 3) dimension reduction by parcellation of the brain using an atlas; 4) averaging the time signal across grey matter voxels contained in each region; 5) regressing out nuisance variables (motion correction parameters, white matter, CSF and global signal) and 6) low pass filtering of the resulting fMRI time series. The set of network nodes is defined by the number of regions contained in the specific parcellation of the brain. The edges are estimated using some measure of associations between each pair of regions such as the Pearson correlation coefficient.

After the correlation matrix containing all these pair-wise correlations has been estimated, building the network requires a threshold to define the set of connected edges of the network. The usual practice is to repeat the graph theory

*Address correspondence to this author at the Department of Biostatistical Sciences, Wake Forest University Health Sciences, Winston-Salem, NC, 27157 USA; Tel: 336- 716 8309; Fax: 336-716-6427;

E-mail: casanova@wfubmc.edu analyses for different choices of the threshold or other criteria that aim at keeping some feature of the network constant across subjects, such as network cost [10], average degree [13] or characteristic path length [17]. However, it is not clear that an optimum approach exists.

We use machine learning methods to perform group analyses of R-fMRI brain networks avoiding the threshold selection problem mentioned above. The central idea is to use the full set of correlations as input variables for a large scale classification algorithm. Our work is related to previous research developed by [18-20] who implemented different feature selection techniques to locate discriminative graph edges (correlations) when analyzing R-fMRI and block design fMRI data respectively. We used here two different ensemble methods: 1) Random Forests (RF) [21] and 2) a new ensemble method based on least angle shrinkage and selection operator (lasso) regression.

$\mathrm{RF}$ has several properties that explain its increasing popularity in bioinformatics [22-28]: 1) it can be used when there are more variables than observations; 2) RF can deal with two class and multi-class problems; 3) it does not overfit; 4) RF can handle mixtures of categorical and continuous variables; 5) RF contains a built-in crossvalidation method using out of the bag (OOB) samples and 6) it provides continuous measures of importance rather than binary responses.

Additionally, we introduce here an ensemble method based on lasso regression that takes advantage of lasso's sparsity property [29] for embedded feature selection. Previously, ensembles of ridge regression and least square classifiers based on subsampling have been proposed and applied to different problems [30,31]. We also propose an index for scoring the importance of a variable, which is a natural consequence of lasso's sparsity property. Since lasso regression forces many coefficients to be zero for each individual classifier, the frequency with which each variable 
is contained in the model across all members of the ensemble can be computed and taken as a measure of its relevance. This is a similar concept as the relevance counts proposed by [32]. The main differences are: 1) they computed the scores of relevance during the testing phase of a two-fold $\mathrm{CV}$, a process that they repeated 100 times while we here computed the scores based on subsampled versions of the data set and the ensemble learning approach; 2) they were working on penalized logistic regression while we are using penalized linear regression and 3) we use a very efficient implementation of lasso regression based on the coordinate descent technique provided by the GLMNET library $[33,34]$ that allows the full data space computations to be performed in a time-efficient manner. This allows us to implement permutation testing to determine significance not only of classification accuracy but also of the selected features.

Differently from previous work we don't use any feature reduction steps prior to classification, such as univariate filtering [18, 19], but we operate directly with the entire input space (the full set of correlations). We applied these two methods to resting state data from the Connectome Project [35] in order to study differences in functional connectivity across sex. Gender differences are thought to exist across a variety of cognitive domains, and have been demonstrated consistently in studies investigating sexual dimorphism of cognitive ability during performance of specific tasks [36-38]. For example, superior performance has been reported in women on tasks of verbal fluency and emotion-oriented memory [39-43], whereas superior performance has been reported in men on tasks of mental rotation and visuospatial ability [44-46]. Such task performance may reflect innate gender differences in the functional organization of the brain, and have been the focus of investigations evaluating asymmetries in functional cerebral organization between males and females using a variety of neuroimaging techniques, including PET, SPECT, and task-related bloodoxygen-level dependence (BOLD) fMRI [47]. In particular, gender-associated differences in brain activation have been reported in frontal, temporal, parietal, occipital, and thalamic regions, as well as in insular and cingulate cortex, using multiple different fMRI paradigms [48-54]. Unlike these prior studies investigating task-specific gender differences in functional brain activity, the present study is one of a few to date that aims to evaluate key differences between males and females in brain network connectivity, and is the first to identify specific gender-discriminative edges and their associated nodes.

\section{MATERIALS AND METHODOLOGY}

\section{Resting State Data}

\section{Participants}

R-fMRI BOLD data were acquired from the 1000 Functional Connectome Project (http://www.nitrc.org/projects/ fcon_1000) (FCP), which is a publicly accessible database [35]. Specifically, data from 148 subjects (74 female and 74 male; matched by age (21 years old) recruited as part of larger studies conducted in Beijing China were used. Subjects were reported to be healthy and without history of head injury, psychiatric or neurological disorder $[55,56]$. All subjects provided written informed consent approved by the Institutional Review Board of the State Key Laboratory of Cognitive Neuroscience and Learning, Beijing Normal University $[55,56]$.

\section{MRI Scanning}

The 148 Beijing FCP subjects were scanned in a Siemens 3.0 Tesla Trio MR scanner for collection of T1-weighted structural anatomic (MP-RAGE) MRI and resting-state fMRI-BOLD data. Resting state fMRI was acquired using echo planar imaging $(\mathrm{EPI})(\mathrm{TR}=2000 \mathrm{~ms}$; $\mathrm{TE}=30 \mathrm{~ms}$; field of view $=200 \times 200 \mathrm{~mm}^{2}$; matrix size $=64 \times 64,33$ axial slices, $3 \mathrm{~mm}$ thickness, $0.6 \mathrm{~mm}$ gap; voxel resolution $=3.125$ $\mathrm{mm} \times 3.125 \mathrm{~mm} \times 3.6 \mathrm{~mm})$. The subjects performed no task, but were asked to keep their eyes closed for the 7.5 minute R-fMRI scan [55, 56].

\section{Image Processing}

All data were motion-corrected and normalized to a standard template using the version 8 of the statistical parametric mapping software(SPM8) [57]. The structural T1-weighted images were segmented into grey matter and cerebrospinal fluid (CSF) using the SPM8 new segment tool. Segmentation maps were resampled to the space of the normalized functional MRI data for use as masks in the postprocessing. Preprocessing included regression of motion parameters, nuisance signals (white matter and ventricular mean signal from the segmentation maps) and global signal, followed by band-pass filtering at $0.01-0.1 \mathrm{~Hz}$ to isolate the low-frequency fluctuations characteristic of resting connectivity. Data was then parcellated into 116 regions using the Automatic Anatomical Labeling (AAL) atlas [58], as implemented by the wfu_pickatlas [59] and masked with the grey matter segmentation map to limit the nodes to grey matter structures. This resulted in an averaged fMRI time series of 116 regions (nodes) for each subject, which was used for subsequent connectivity analysis. To establish the presence of functional connectivity between the parcellated regions (nodes), the Pearson correlation was computed between all pairs of node time series to generate a $116 \times 116$ correlation matrix $(\mathrm{Cij})$ for each subject.

\section{Classification of Brain Networks}

The classification of R-fMRI brain networks problem can be formulated as the detection of discriminative edges between two groups of weighted brain networks. For each subject the set of nodes is defined by the different brain regions of interest (ROIs) from the specific parcellation (AAL atlas in our case) and the edge weights are given by the pair-wise Pearson correlation coefficients between brain regions (average R-fMRI signal) contained in the correlation matrix. Because this matrix is symmetric the feature vector (or sample) corresponding to each subject will be composed of the correlations contained in the upper triangle of the subject's correlation matrix that are extracted and vectorized. The matrix of predictors is formed by stacking the feature vectors corresponding to all individuals participating in the analysis. Each subject is also assigned a label identifying the participants' sex. This information is then provided to the software implementing the two classifiers. 


\section{Random Forests}

RF belongs to the category of the so called ensemble methods for classification because a committee of learners (trees in this case) is generated and each one casts a vote for the predicted label of a given instance. The trees are built using the classification and regression trees methodology (CART) [60]. In constructing the ensemble of trees, RF uses two types of randomness: first each tree is grown using a bootstrapped version of the training data. A second level of randomness is added when growing any given tree by selecting a random sample of predictors at each node to choose the best split. The number of predictors selected at each node and the number of trees in the ensemble are the two main parameters of the RF algorithm. The RF developers have reported that the method does not require much tuning of these parameters and that the default values often produce good results for many problems [21]. Once the forest is built, assigning a new instance to a class is accomplished by combining the trees using majority vote. As a result of using a bootstrap sampling of the training data, around one third of the samples are left out when building each tree. These are the so called out-of-bag (OOB) samples that can be used to assess the performance of the classifier and also to build measures of importance. In this work we will use the permutation importance score. The importance of a variable is evaluated by estimating the increase in prediction error occurring when the OOB data for that variable is permuted while others are left unchanged. The necessary calculations are carried out tree by tree as the random forest is constructed [61]. We used the $\mathrm{R}$ package randomForest [61] with the default parameters for the number of trees (ntree $=500)$ and the number of predictors analyzed at each node to find the best split $(m t r y=\sqrt{p})$.

\section{Lasso Regression}

Lasso regression was introduced by replacing the $\mathrm{L}_{2}$ penalty typical of Tikhonov regularization and ridge regression [62,63] by a $\mathrm{L}_{1}$ penalty [29]. Lasso regression in our case is described by

$$
\min _{\beta_{0}, \beta \in R^{p+1}} \frac{1}{N} \sum_{i=1}^{N}\left(y_{i}-\beta_{0}-x_{i}^{T} \beta\right)^{2}+\lambda\|\beta\|_{1}
$$

where $N$ is the number of samples (148 subjects in our case), $x_{i} \in R^{p}$ is the $\mathrm{i}^{\text {th }}$ sample or feature vector containing the correlations entering the analysis (all elements of the upper triangle of the correlation matrix), $y_{i} \in\{-1,1\}$ is the $\mathrm{i}^{\text {th }}$ label ( -1 female and 1 male), $M=116$ is the number of nodes (from the AAL atlas parcellation), $p=\frac{M(M-1)}{2}=6670$ is the number of correlations (edges), $\beta_{0}, \beta$ are the parameters of the model, and $\lambda$ is the regularization parameter. Lasso is characterized by its sparsity meaning that many of the coefficients will be forced to be zero performing this way a kind of embedded feature selection.

We solve the problem described in Eq. (1) using the GLMNET library [33, 64] which uses a very efficient optimization technique called coordinate descent [65]. This regularization scheme initially developed for regression can be adapted to binary classification problems by using the classification rule $\hat{y}=\operatorname{sign}($ fitted value) where $\hat{y}$ is the estimated label $[66,67]$.

\section{Estimation of Regularization Parameter $\lambda$}

To estimate the optimal values of the regularization parameter we combine a scheme of two nested crossvalidations $(\mathrm{CV})$ with grid search to avoid upward bias in the estimation of accuracy [68-71]. We implemented an external $\mathrm{K}_{1}$-fold CV where at each step we leave one fold for testing and the $\mathrm{K}_{1}-1$ remaining for training and validation. These last two procedures are implemented by using a nested $\mathrm{K}_{2}$ fold CV. We divide the $\mathrm{K}_{1}-1$ folds into $\mathrm{K}_{2}$ folds and we leave one fold for validation and $\mathrm{K}_{2}-1$ for training combined with a grid search to determine the optimal parameters. The grid we used in our analyses was $\lambda=1000,500,200,150,125,100$, $10,1,0.1$. At each grid point the classifier is trained using the training data and its performance is assessed using the fold left for validation by estimating the classification accuracy. We select the regularization parameters that produce maximum average accuracy across the $\mathrm{K}_{2}$ folds of the internal $\mathrm{CV}$ procedure. The classifier is then retrained using the whole data in the $\mathrm{K}_{1}-1$ folds left for training and validation and the selected optimal regularization parameters. The classifier's generalization capability is then evaluated by computing the classification accuracy using the fold originally left for testing in the external $\mathrm{CV}$. This is repeated $\mathrm{K}_{1}$ times and the average classification accuracy is computed and reported in our figures. Finally, the classifier weights are computed using the whole data set and the average values of the selected regularization parameters across the $\mathrm{K}_{1}$ folds. In our analyses we used $\mathrm{K}_{1}=5$ and $\mathrm{K}_{2}=5$.

\section{Ensemble of Lasso Regression Classifiers}

We build our ensemble by training $N_{c}$ lasso regression classifiers according to the methodology presented above (Eq. (1), CV procedure, etc) using for each member of the ensemble a subsampled version of the data. The subsampling is carried out by selecting at random from each class the same number of samples. This means that the number of classifiers included in the ensemble $\left(N_{c}\right)$ and the fraction of subsampled samples (FS) are parameters of this algorithm. There are two sources of randomness associated with this ensemble: 1) Random subsampling and 2) Random partition of the subsampled data to carry out cross-validation. We use the average classification accuracy across the $N_{c}$ classifiers as a measure of performance of the ensemble. Building ensembles with sparse classifiers allows us to introduce importance measures for each variable based on the sparsity property. Given a set of $N_{c}$ sparse classifiers composing the ensemble we can compute the frequency that each variable enters the model across the $N_{c}$ members of the ensemble. We used this frequency as an importance score for the variable. Additionally, we compute the mean values of the coefficients associated with each edge across the members of the ensemble. The sign of the coefficients mean value associated to each edge (correlation) allows us to determine the edges associated to classification as men or women. 


\section{Permutation Testing}

Our implementation of the ensemble is based on a very time efficient methodology allowing us to implement permutation testing. In the neuroimaging literature, permutation testing is often used to assess significance of classification accuracy [72-74]. Here we also use it to evaluate significance of feature selection by combining it with the importance score of each variable. Briefly, we recompute the ensemble $N_{p}=1000$ times with permuted labels. In each case we keep track not only of the values of the classification accuracy but also of the maximum values of the importance scores. The latter permits us to evaluate the significance of the observed scores and of the selected features. We use similar permutation tests to perform feature selection and to evaluate the significance of the results produced by the RF analysis. Specifically, we use the permutation importance score [21] to evaluate significance of feature selection.

\section{Software and Hardware Platforms}

Our software implementation is based on MATLAB in which the GLMNET library is called using a freely available MATLAB wrapper developed by Hui Jiang (http://wwwstat.stanford.edu/ tibs/glmnet-matlab/). The computations were performed on a DELL computer system with eight 2.66 $\mathrm{GHz}$ CPUs and 16 GB of RAM memory. Parallel programming based on the MATLAB parallel computing toolbox was used to speed up computations.

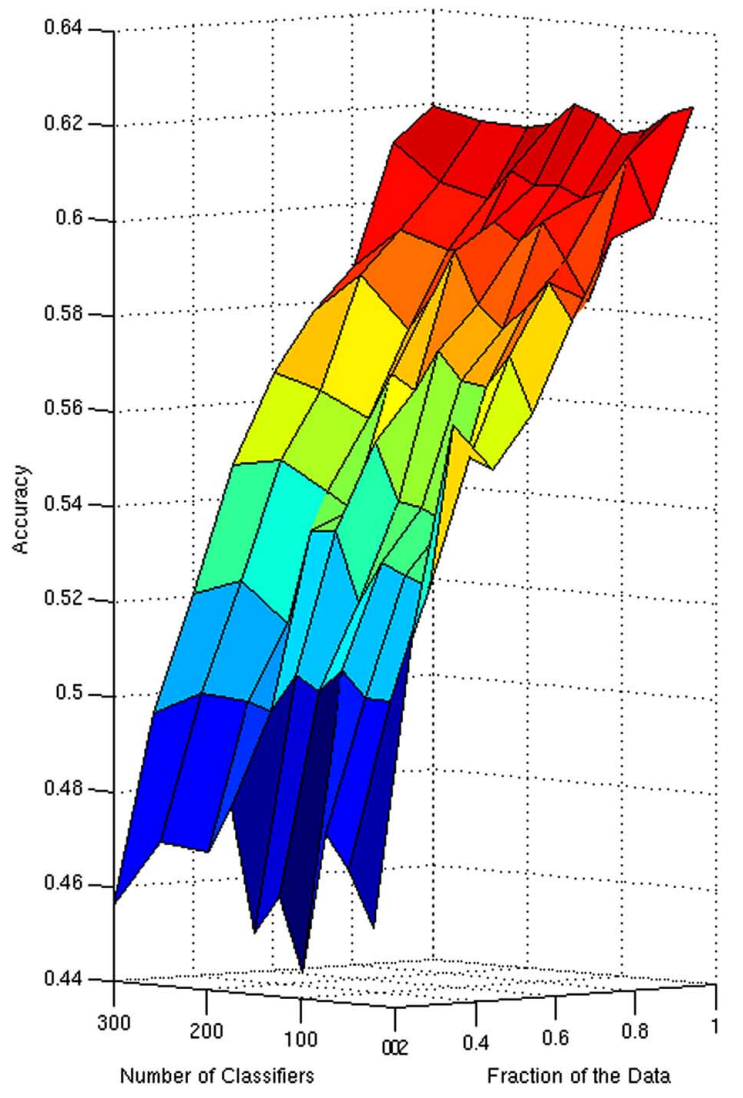

\section{RESULTS}

Since the number of classifiers in the ensemble and the fraction of the samples used during subsampling are additional parameters of the ensemble of lasso regression classifiers (ELRC), before carrying out the final analysis of our data we explored the performance of the algorithm for different values of these two parameters (Fig. 1). The best performances in terms of the average classification accuracy are achieved when all the samples $(\mathrm{FS}=1)$ are used to estimate each member of the ensemble (Fig. 1, left panel). The number of classifiers in the ensemble seems to have little influence on the final result, which approximates $62 \%$. Additionally, the sparser set of selected features also correspond to the choice of FS $=1$. In this case for all values of $N_{c}$, the number of selected features (edges) was the same.

For the final analysis of the R-fMRI data set we fixed $N_{c}=100$ and FS $=1$. The observed overall average accuracy was $62.3 \%$ computed as the average classification accuracy across the members of the ensemble. The permutation test of the overall classification accuracy and the importance scores for the variables were both significant at $p<0.001$ and $p$ $<0.05$. The discriminative edges associated with classification as women and men are described in Tables $\mathbf{1}$ and $\mathbf{2}$, respectively. The brain regions (nodes) associated with the discriminative edges in women (upper row) and men (bottom row) are shown in Fig. (2). The RF analysis produced $65.4 \%$ classification accuracy which was significant $(\mathrm{p}<0.001)$

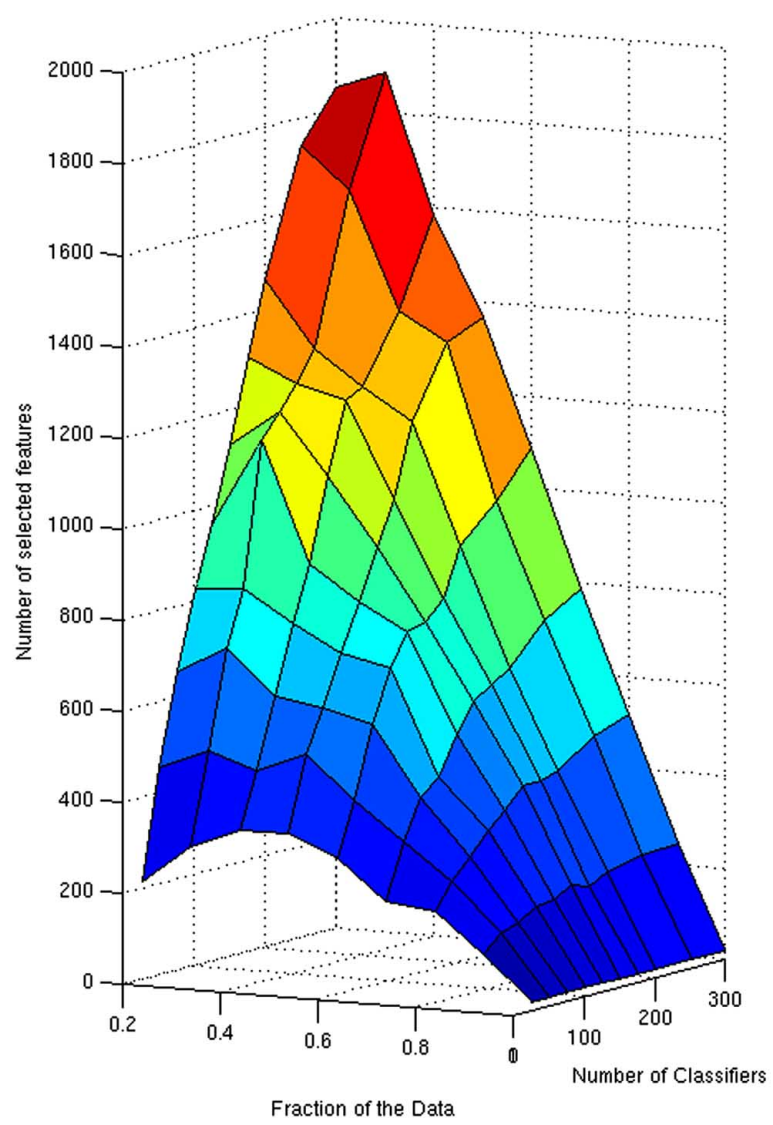

Fig. (1). Surface plots displaying the resulting classification accuracy (left panel) and the number of selected features (right panel) for different choices of the number of classifiers and the fraction of the data to be subsampled. 


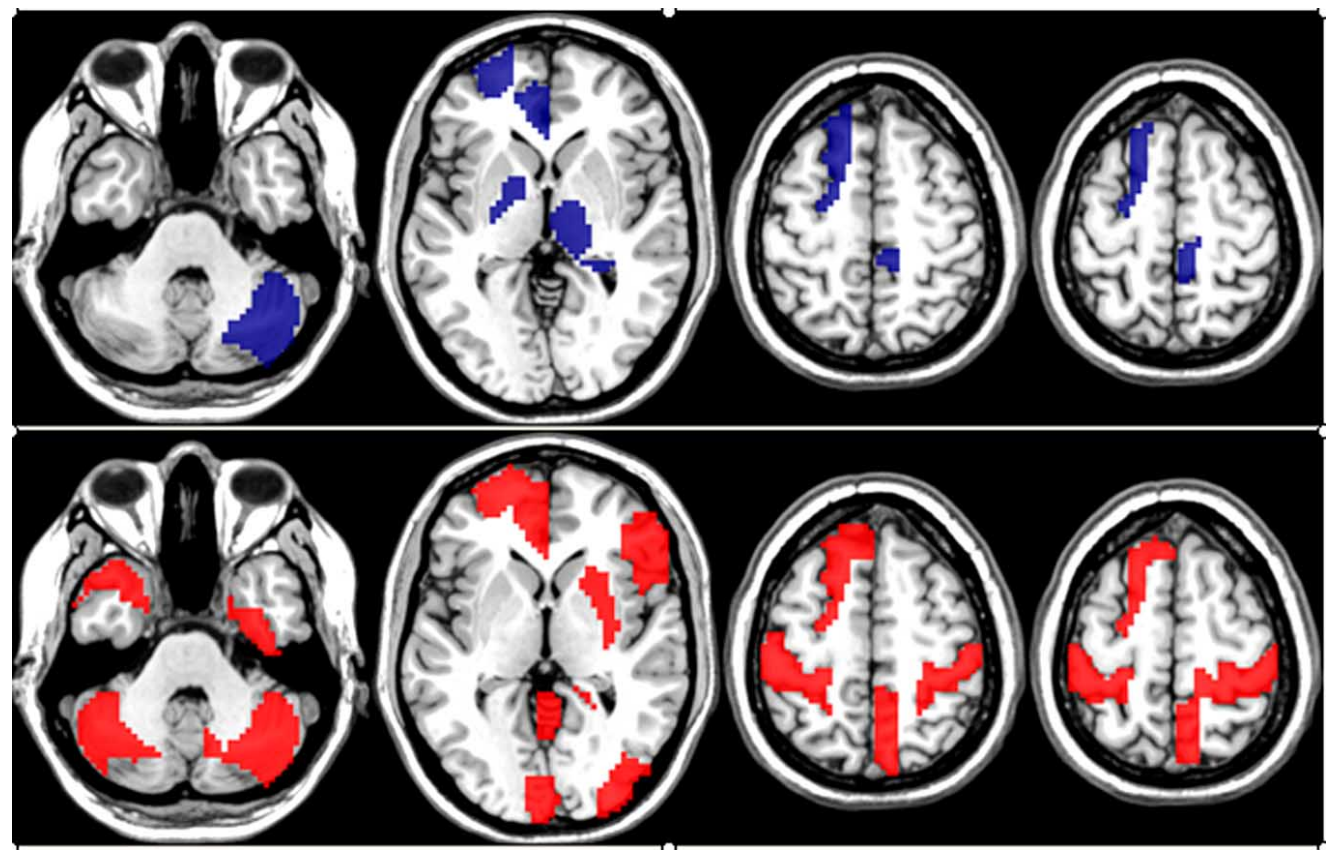

Fig. (2). Network nodes (brain regions) associated with the more discriminative edges generated by the ensemble of lasso regressors method. Top row: nodes related to the discriminative edges associated with classification as female (blue color). Bottom: nodes related to the discriminate edges associated with classification as males (red color).

Table 1. Nodes Connecting Discriminative Edges Associated with Classification as Female According to the ELRC Analysis

\begin{tabular}{|c|c|c|c|}
\hline \multicolumn{2}{|c|}{ AAL Nodes Numbers } & Brain Area 1 & Brain Area 2 \\
\hline \hline 2 & 92 & Frontal_Superior Left & Cerebellum_Crus1_R \\
\hline 28 & 53 & Rectus_R & Occipital_Inf_L \\
\hline 31 & 38 & Cingulum_Ant_L & Hipoccampus_R \\
\hline 33 & 78 & Cingulum_Mid_L & Thalamus_R \\
\hline 33 & 112 & Cingulum_Mid_L & Vermis_6 \\
\hline 70 & 75 & Paracentral_Lobule_R & Pallidum_L \\
\hline
\end{tabular}

using a permutation test. A permutation test applied to permutation importance RF scores produced a total of four discriminative edges $(p<0.05)$. The list of these edges is presented in Table $\mathbf{3}$ and the related anatomical areas are shown in Fig. (3). Because RF is a highly nonlinear classifier it is not possible to provide the information about the association of discriminative edges and sex.

The nodes connecting discriminative edges in women localized to the cingulate gyrus, left frontal lobe, deep grey structures (basal ganglia, thalami), and right cerebellum. In men, these regions localized predominantly to the cingulate gyrus, as well as bilateral sensorimotor cortices, bilateral cerebellum, and left frontal lobe.

\section{DISCUSSION}

In this work we have applied two different ensemble classification methods to analyze differences in functional connectivity across gender. RF is a well know technique in bioinformatics while the ELRC has been introduced here. This last approach combines the sparsity property of lasso

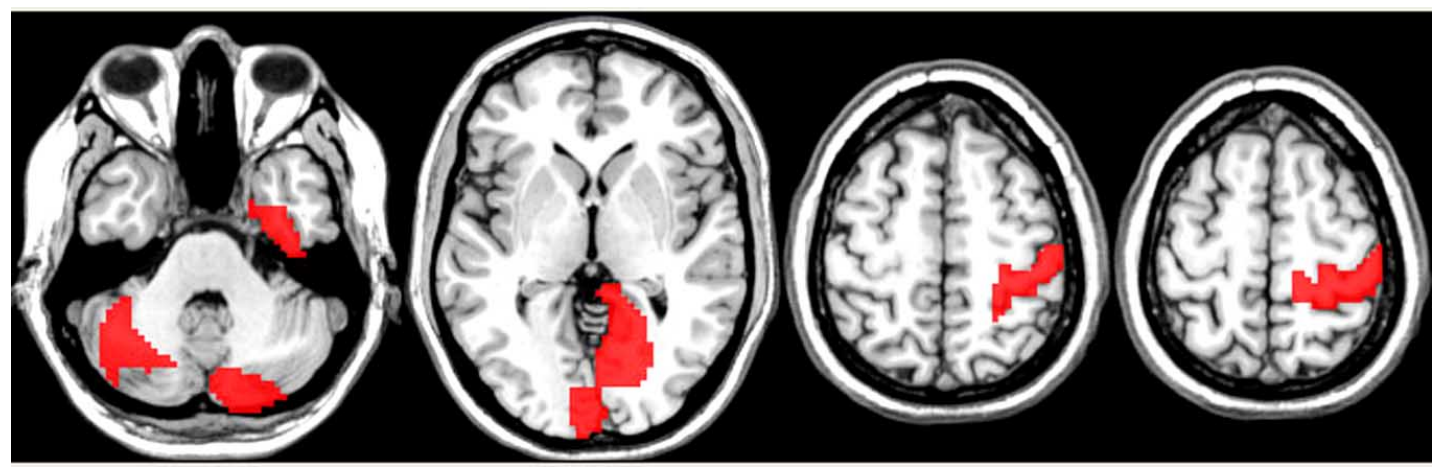

Fig. (3). Network nodes associated with discriminative edges using Random Forest Analysis. No additional information regarding the nature of the associations is provided by the RF analysis. In red color are shown the nodes associated to discriminative edges that were significantly $(\mathrm{p}<0.05$ corrected) relevant for classification in general. 
Table 2. Nodes Connecting Discriminative Edges Associated with Classification as Males According to the ELRC Analysis

\begin{tabular}{|c|c|c|c|}
\hline \multicolumn{2}{|c|}{ AAL Nodes Numbers } & Brain Area 1 & Brain Area 2 \\
\hline \hline 3 & 52 & Frontal_Superior Left & Putamen_R \\
\hline 12 & 74 & Frontal_Inf_Oper_R & Cingulum_Ant_L \\
\hline 14 & 31 & Frontal_Inf_Tri_R & Temporal_Pole_Mid_L \\
\hline 23 & 87 & Frontal_Sup_Medial_L & Postcentral_L \\
\hline 33 & 57 & Cingulum_Mid_L & Cerebellum_8_R \\
\hline 33 & 58 & Cingulum_Mid_L & Fusiform_R \\
\hline 35 & 104 & Cingulum_Post_L & Cerebellum_Crus1_L \\
\hline 40 & 56 & Parahippocampal_R & Cerebellum_Crus1_R \\
\hline 43 & 91 & Calcarine_L & Precuneus_R \\
\hline 43 & 92 & Calcarine_L & Vermis_4_5 \\
\hline 45 & 68 & Cuneus_L & Vermis_3 \\
\hline 110 & 111 & & \\
\hline
\end{tabular}

Table 3. Nodes Connecting Discriminative Edges Produced by RF Analysis

\begin{tabular}{|c|c|c|c|}
\hline \multicolumn{2}{|c|}{ AAL Nodes Numbers } & Brain Area 1 & Brain Area 2 \\
\hline \hline 33 & 58 & Cingulum_Mid_L & Postcentral_R \\
\hline 40 & 56 & Parahippocampal_R & Fusiform_R \\
\hline 43 & 94 & Calcarine_L & Cerebellum_Crus2_R \\
\hline 48 & 91 & Lingual_R & Cerebellum_Crus1_L \\
\hline
\end{tabular}

regression with the concept of ensemble learning. The former will perform feature selection by forcing many predictors' coefficients to be zero while the latter will allow defining importance scores for each variable by estimating the frequency of its appearance across all the models defining the members of the ensemble. These machine learning methodologies allow performing group analyses of brain networks without previous selection of thresholds.

The final result in both cases is a set of edges that carry discriminative information between the two groups of networks. In the case of the ELRC we used a very fast implementation of lasso regression provided by the library GLMNET that allowed us to use permutation testing not only for assessing the significance of accuracy but also for the importance of selected features.

The results produced by both methodologies were consistent in terms of the levels of classification accuracy and statistical significance. While the levels of classification accuracy were very similar the statistical significance of the results in both cases was the same. Despite very different underlying mechanisms, both methods detected common edges and nodes as more discriminative which are the more robust findings in this study. Our results suggest that the ELRC detected more discriminative edges than RF. Simulations necessary to confirm this finding were out of the scope of this work, as they are very time consuming and our focus was mainly on finding sex differences in R-fMRI brain networks. In addition, ELRC provides useful information about the association of the detected edges to classification as male or female. But this is a general advantage of linear classifiers over non-linear ones. Very often non-linear classifiers in high dimensional problems do not produce improvements while being at the same time more difficult to interpret because the linear classifiers generate weights for each predictor that can be used as a measure of their importance within the estimated model $[72,75]$. Because RF is a highly non-linear classifier it does not provide this type of information.

There are some limitations in the ELRC methodology. There is a lack of a method to select optimal values of the number of classifiers of the ensemble $\left(N_{c}\right)$ and the fraction of samples used to generate each member of the ensemble. This is a common problem with previous approaches [30, $31]$. Here via trial and error we have chosen the value of the fraction of samples that leads to higher values of average overall accuracy computed across all members of the ensembles. This amounts to performing lasso regression $N_{c}$ times with different $\mathrm{CV}$ partitions.

Although RF is a highly nonlinear classifier and, therefore, unable to provide information about the association of discriminative edges and sex, many of the discriminative edges identified using this technique overlap with discriminative edges associated with classification as male according to the ELRC analysis. Specifically, both the RF and ELRC 
methods identify key discriminative edges between: 1 . left middle cingulum and right postcentral gyrus, 2. right parahippocampal gyrus and right fusiform gyrus, as well as, 3 . left calcarine fissure and right crus of the cerebellum (Tables 2 and 3). These gender-discriminative differences are consistent with findings described in other studies investigating sexual dimorphism of network connectivity using graph theoretical methods [76, 77]. Wang and colleagues demonstrated lower nodal efficiency in females compared to males in the left middle cingulum and right parahippocampal gyrus. In addition, Tian and colleagues identified the left middle cingulate gyrus as an important hub node in males and females [77]. The ELRC method identified additional nodes connected by gender discriminative edges in the present investigation that correspond to nodes that Wang et al., demonstrated to have gender-associated differences in efficiency, including frontal (left middle frontal gyrus), temporal (right superior temporal gyrus), and limbic/paralimbic regions (left hippocampus, right hippocampus, and left amygdala) [76]. It is possible that gender-related differences in nodal efficiency [76] may reflect sexually dimorphic variability in the nodes with which they connect, as demonstrated in the present study (Tables $\mathbf{1}$ and $\mathbf{2}$ ). It is possible that differences in functional connectivity between males and females may also be related to gender-related differences in regional connectivity between hub nodes [77].

In the present study, males demonstrated a greater proportion of gender-discriminative edges associated with sensory, motor and association regions than females, which may be related to known male gender performance differences in visuospatial tasks [44-46]. As compared to males, females demonstrated a greater proportion of gender-discriminative edges associated with limbic regions, although both males and females had discriminative limbic-associated edges. In particular, there were distinct differences between males and females in specific limbic areas associated with gender-discriminative edges. For example, males but not females had a discriminative edge associated with the posterior cingulum and parahippocampal gyrus, which are known to be involved in visuospatial processing and formation of spatial memories, respectively [78, 79] (Tables 1 and 2). Interestingly, men have demonstrated greater BOLD activation than women in posterior cingulum and parahippocampal gyrus during performance of visuospatial navigation fMRI tasks $[80,81]$.

Other limbic areas, such as the anterior and middle cingulum, were identified in the present study as important nodes transmitting discriminative edges in both males and females (Tables $\mathbf{1}$ and 2). Edges associated with the anterior and middle cingulum may be gender-discriminative because of the nodes with which they connect, which differ between males and females. For example, the anterior cingulum, known to be involved in affect processing [78], is connected by a gender-discriminative edge to the right hippocampus in females, but to the triangular inferior frontal gyrus in males (Tables $\mathbf{1}$ and 2). Similarly, the middle cingulum, which is known to be involved in response selection [78], is connected by gender-discriminative edges to the vermis and right thalamus in females, but to the left and right posteriorcentral gyrus in males (Tables $\mathbf{1}$ and 2). It is possible that these gender-associated differences in anterior and middle cingulate connectivity may contribute to known differences in cingulate BOLD activation associated with emotionprocessing tasks between men and women that are correlated with fMRI task performance [82-84].

Taken together, these data suggest that gender differences in brain function may be related to sexually dimorphic regional connectivity between specific critical nodes via gender-discriminative edges. Furthermore, such differences may be related to performance advantages of females on tasks such as verbal memory and selective attention [39-43], and males on tasks of mental rotation and visuospatial ability [44-46]. More work is necessary, however, to further investigate these possibilities.

\section{CONCLUSION}

We have applied machine learning methodologies to the analysis of functional connectivity based on resting state fMRI. These methods can analyze weighted brain networks and detect the specific set of edges that are informative for differences between groups of brain networks. When applied to R-fMRI data from a group of normal males and females, we were able to identify gender-discriminative edges that may be related to sexually dimorphic regional connectivity between specific critical nodes. Such methods may be more broadly applied in the future to identify discriminative edges in the brain for between-group differences that have important clinical implications.

\section{ACKNOWLEDGEMENTS}

We thank Susan Shear for computer programming support. This work is funded in part by the NIH Supplement \# HHSN268200464221C added to the N01-WH-4-4221 grant.

\section{CONFLICT OF INTEREST}

None declared.

\section{REFERENCES}

[1] Biswal B, Yetkin FZ, Haughton VM, et al. Functional connectivity in the motor cortex of resting human brain using echo-planar MRI. Magn Reson Med 1995; 34(4): 537-41.

[2] Cordes D, Haughton VM, Arfanakis K, et al. Frequencies contributing to functional connectivity in the cerebral cortex in "resting-state" data. AJNR Am J Neuroradiol 2001; 22(7): 1326-33.

[3] Cordes D, Haughton VM, Arfanakis K, et al. Mapping functionally related regions of brain with functional connectivity MR imaging. AJNR Am J Neuroradiol 2000; 21(9): 1636-44.

[4] Kiviniemi V, Kantola J, Jauhiainen J, Tervonen O, et al. Comparison of methods for detecting nondeterministic BOLD fluctuation in fMRI. Magn Reson Imaging 2004; 22(2): 197-203.

[5] Bullmore E, Barnes A, Bassett DS, et al. Generic aspects of complexity in brain imaging data and other biological systems. Neuroimage 2009; 47(3): 1125-34.

[6] Bullmore E, Sporns O. Complex brain networks: graph theoretical analysis of structural and functional systems. Nat Rev Neurosci 2009; 10(3): 186-98.

[7] Watts DJ, Strogatz SH. Collective dynamics of 'small-world' networks. Nature 1998; 393(6684): 440-2.

[8] Barabasi AL, Albert R. Emergence of scaling in random networks. Science 1999; 286(5439): 509-12.

[9] Eguiluz VM, Chialvo DR, Cecchi GA, Baliki M, Apkarian AV. Scale-free brain functional networks. Phys Rev Lett 2005; 94(1): 018102 . 
[10] Achard S, Bullmore E. Efficiency and cost of economical brain functional networks. PLoS Comput Biol 2007; 3(2): e17.

[11] Achard S, Salvador R, Whitcher B, Suckling J, Bullmore E. A resilient, low-frequency, small-world human brain functional network with highly connected association cortical hubs. J Neurosci 2006; 26(1): 63-72.

[12] Salvador R, Suckling J, Coleman MR, et al. Neurophysiological architecture of functional magnetic resonance images of human brain. Cereb Cortex 2005; 15(9): 1332-42.

[13] van den Heuvel MP, Mandl RC, Kahn RS, et al. Small-world and scale-free organization of voxel-based resting-state functional connectivity in the human brain. Neuroimage 2008; 43(3): 528-39.

[14] Chen ZJ, He Y, Rosa-Neto P, et al. Revealing modular architecture of human brain structural networks by using cortical thickness from MRI. Cereb Cortex 2008; 18(10): 2374-81.

[15] He Y, Chen ZJ, Evans AC. Small-world anatomical networks in the human brain revealed by cortical thickness from MRI. Cereb Cortex 2007; 17(10): 2407-19.

[16] Iturria-Medina Y, Sotero RC, Canales-Rodríguez EJ, AlemánGómez Y, Melie-García L. Studying the human brain anatomical network via diffusion-weighted MRI and graph theory. Neuroimage 2008; 40(3): 1064-76.

[17] Hayasaka S, Laurienti PJ. Comparison of characteristics between region-and voxel-based network analyses in resting-state fMRI data. Neuroimage 2010; 50(2): 499-508.

[18] Craddock RC, Holtzheimer PE, Hu XP, Mayberg HS. Disease state prediction from resting state functional connectivity. Magn Reson Med 2009; 62(6): 1619-28.

[19] Richiardi J, Eryilmaz H, Schwartz S, Vuilleumier P, Van De Ville D. Decoding brain states from fMRI connectivity graphs. Neuroimage 2011; 56: 616-26.

[20] Chen G, Ward BD, Xie C. Classification of Alzheimer disease, mild cognitive impairment, and normal cognitive status with large-scale network analysis based on resting-state functional MR imaging. Radiology 2011; 259(1): 213-21.

[21] Breiman L. Random Forests. Mach Learn 2001; 45: 5-32.

[22] Diaz-Uriarte R, Alvarez de Andres S. Gene selection and classification of microarray data using random forest. BMC Bioinformatics 2006; 7: 3 .

[23] Lunetta KL, Hayward LB, Segal J, Van Eerdewegh P. Screening large-scale association study data: exploiting interactions using random forests. BMC Genet 2004; 5(1): 32 .

[24] Bureau A, Dupuis J, Falls K. Identifying SNPs predictive of phenotype using random forests. Genet Epidemiol 2005; 28(2): $171-82$.

[25] McKinney BA, Crowe JE, Guo J, Tian D. Machine learning for detecting gene-gene interactions: a review. Appl Bioinformatics 2006; 5(2): 77-88.

[26] Lehmann C, Koenig T, Jelic V, et al. Application and comparison of classification algorithms for recognition of Alzheimer's disease in electrical brain activity (EEG). J Neurosci Methods 2007; 161(2): 342-50.

[27] Tripoliti EE, Fotiadis DI, Argyropoulou M. A supervised method to assist the diagnosis of Alzheimer's disease based on functional magnetic resonance imaging. Conf Proc IEEE Eng Med Biol Soc 2007; 2007: 3426-9.

[28] Casanova R, Espeland MA, Goveas JS, et al. Application of machine learning methods to describe the effects of conjugated equine estrogens therapy on region-specific brain volumes. Magn Reson Imag 2011; 29(4): 546-53.

[29] Tibshirani R. Regression Shrinkage and Selection via the Lasso. J. R. Statist Soc B 1996; 58(1): 267-88.

[30] Torkkola K, Tuv E. Ensembles of Regularized Least Squares Classifiers for High Dimensional Problems, in Feature Extraction: Foundations and Applications, I. Guyon, et al., Editors. 2006, Springer.

[31] Nikulin V, McLachlan GC. Classification of imbalanced marketing data with balanced random sets. JMLR: Workshop Conf Proc 2009; 7: 89-100.

[32] Shevade K, Keerthi S. A simple and efficient algorithm for gene selection using sparse logistic regression. Bioinformatics 2003; 19: 2246-53.

[33] Friedman J, Hastie T, Tibshirani R. Regularization paths for generalized linear models via coordinate descent. J Statist Software 2010; 33(1): 1-22.
[34] Friedman J, Hastie T, Tibshirani R. glmnet: Lasso and Elastic Net Regularized Generalized Linear Models. R package version 1.1-4 2009.

[35] Biswal BB, Mennes M, Zuo XN, et al. Toward discovery science of human brain function. Proc Natl Acad Sci USA 2010; 107(10): 4734-9.

[36] Kimura D. Sex, sexual orientation and sex hormones influence human cognitive function. Curr Opin Neurobiol 1996; 6(2): 259-63.

[37] Nicholson KG, Kimura D. Sex differences for speech and manual skill. Percept Mot Skills 1996; 82(1): 3-13.

[38] Wegesin DJ. A neuropsychologic profile of homosexual and heterosexual men and women. Arch Sex Behav 1998; 27(1): 91108.

[39] Hyde JS, Linn MC. Gender differences in verbal-ability - a metaanalysis. Psychol Bull 1988; 104(1): 53-69.

[40] Davis PJ. Gender differences in autobiographical memory for childhood emotional experiences. J Pers Soc Psychol 1999; 76(3): 498-510.

[41] Thayer JF, Johnsen BH. Sex differences in judgement of facial affect: a multivariate analysis of recognition errors. Scand J Psychol 2000; 41(3): 243-6.

[42] Canli T, Desmond JE, Zhao Z, Gabrieli DE. Sex differences in the neural basis of emotional memories. Proc Natl Acad Sci U S A 2002; 99(16): 10789-94.

[43] Rahman Q, Wilson GD, Abrahams S. Sex, sexual orientation, and identification of positive and negative facial affect. Brain Cogn 2004; 54(3): 179-85.

[44] Linn MC, Petersen AC. Emergence and characterization of sex differences in spatial ability: a meta-analysis. Child Dev 1985 56(6): 1479-98.

[45] Crucian GP, Berenbaum SA. Sex differences in right hemisphere tasks. Brain Cogn 1998; 36(3): 377-89.

[46] Voyer D, Voyer S, Bryden MP. Magnitude of sex differences in spatial abilities: a meta-analysis and consideration of critical variables. Psychol Bull 1995; 117(2): 250-70.

[47] Cosgrove KP, Mazure CM, Staley JK. Evolving knowledge of sex differences in brain structure, function, and chemistry. Biol Psychiatry 2007; 62(8): 847-55.

[48] Shaywitz BA, Haywitz SE, Pugh KR, et al. Sex differences in the functional organization of the brain for language. Nature 1995; 373(6515): 607-9.

[49] Lee TM, Liu HL, Hoosain R. Gender differences in neural correlates of recognition of happy and sad faces in humans assessed by functional magnetic resonance imaging. Neurosci Lett 2002; 333(1): 13-6.

[50] Ragland JD, Coleman AR, Gur RC, Glahn DC, Gur RE. Sex differences in brain-behavior relationships between verbal episodic memory and resting regional cerebral blood flow. Neuropsychologia 2000; 38(4): 451-61.

[51] Weiss E, Siedentopf CM, Hoferet A, et al. Sex differences in brain activation pattern during a visuospatial cognitive task: a functional magnetic resonance imaging study in healthy volunteers. Neurosci Lett 2003; 344(3): 169-72.

[52] Fischer H, Fransson P, Wright CI, Bäckman L. Enhanced occipital and anterior cingulate activation in men but not in women during exposure to angry and fearful male faces. Cogn Affect Behav Neurosci 2004; 4(3): 326-34.

[53] Lee TMC, Liu HL, Chan CC, Fang SY, Gao JH. Neural activities associated with emotion recognition observed in men and women. Mol Psychiatry 2005; 10(5): 450-5.

[54] Bell EC, Willson MC, Wilman AH, Dave S, Silverstone PH. Males and females differ in brain activation during cognitive tasks. Neuroimage 2006; 30(2): 529-38.

[55] Liu D, Yan C, Ren J, Yao L, Kiviniemi VJ, Zang Y. Using coherence to measure regional homogeneity of resting-state FMRI signal. Front Syst Neurosci 2010; 4: 24.

[56] Yan H, Zuo X, Wang D. Hemispheric asymmetry in cognitive division of anterior cingulate cortex: A resting-state functional connectivity study. Neuroimage 2009; 47(4): 1579-89.

[57] Friston KJ, Holmes AP, Worsley KJ, Poline JP, Frith CD, Frackowiak RSJ. Statistical parametric maps in functional imaging: A general linear approach. Hum Brain Mapp 1994; 2(4): 189-210.

[58] Tzourio-Mazoyer N, Landeau B, Papathanassiou D. Automated anatomical labeling of activations in SPM using a macroscopic anatomical parcellation of the MNI MRI single-subject brain. Neuroimage 2002; 15(1): 273-89. 
[59] Maldjian JA, Laurienti P, Burdette J. An automated method for neuroanatomic and cytoarchitectonic atlas-based interrogation of fMRI data sets. Neuroimage 2003; 19(3): 1233-9.

[60] Breiman L, Friedman JH, Olshen RA, Stone CJ. Classification and Regression Trees 1984: Chapman \& Hall/CRC.

[61] Liaw A, Wiener M. Classification and Regression by randomForest. Rnews 2002; 2: 18-22.

[62] Hoerl A. Application of ridge analysis to regression problems. Chem Eng Prog 1962; 58: 54-9.

[63] Tikhonov AN, Arsenin VY. Solution of ill-posed problems. 1977, Washington DC: W.H. Winston.

[64] Friedman J, Hastie T, Tibshirani R. glmnet: Lasso and Elastic Net Regularized Generalized Linear Models. R package version 1.1-4, 2009.

[65] Friedman J, Hastie T, Tibshirani R. Pathwise Coordinate Optimization. Annals Appl Stat 2007; 1(2): 302-32.

[66] Zou H, Hastie T. Regularization and variable selection via the elastic net. J R Statist Soc B 2005; 67: 301-20.

[67] Raizada RD, Tsao FM, Liu HM, Holloway ID, Ansari D, Kuhl PK. Linking brain-wide multivoxel activation patterns to behaviour: Examples from language and math. Neuroimage 2010; 51: 462-71

[68] Cheng CW, Su EC, Hwang JK, Sung TY, Hsu WL. Predicting RNA-binding sites of proteins using support vector machines and evolutionary information. BMC Bioinformatics 2008; 9 (Suppl 12): S6.

[69] Varma S, Simon R. Bias in error estimation when using crossvalidation for model selection. BMC Bioinformatics 2006; 7: 91.

[70] Chu C. Pattern recognition and machine learning for magnetic resonance images with kernel methods, in Wellcome Trust Center for Neuroimaging. 2009, University College London: London.

[71] Ryali S, Supekar K, Abrams DA, Menon V. Sparse logistic regression for whole brain classification of fMRI data. Neuroimage 2010; 51(2): 752-64.

[72] Pereira F, Mitchell T, Botvinick M. Machine learning classifiers and fMRI: a tutorial overview. Neuroimage 2008; 45(Suppl 1): S199-209.

[73] Golland P, Fischl B. Permutation tests for classification: towards statistical significance in image-based studies. Inf Process Med Imag 2003; 18: 330-41.
[74] Ecker C, Marquand A, Mourão-Miranda J. Describing the brain in autism in five dimensions--magnetic resonance imaging-assisted diagnosis of autism spectrum disorder using a multiparameter classification approach. J Neurosci 2010; 30(32): 10612-23.

[75] Hastie T, Tibshirani R, Friedman J. The Elements of Statistical Learning: Data Mining, Inference, and Prediction. Second ed. Springer Series on Statistics. 2009: Springer.

[76] Wang L, Zhu C, He Y, Zhong Q, Zang Y. Gender Effect on Functional Networks in Resting Brain. MIMI, LNCS 2008; 4987: 160-8.

[77] Tian L, Wang J, Yan C, He Y. Hemisphere- and gender-related differences in small-world brain networks: A resting-state functional MRI study. Neuroimage 2010; 54: 191-202.

[78] Vogt BA, Nimchinsky EA, Hof PR. Human cingulate cortex: Surface features, flat maps, and cytoarchitecture. J Comp Neurol 1997; 359: 490-506.

[79] Maguire EA. Hippocampal involvement in human topographical memory: evidence from functional imaging. Philos Trans R Soc Lond B Biol Sci 1997; 352(1360): 1475-80.

[80] Gron G, Wunderlich A, Spitzer M, Tomczak R, Riepe M. Brain activation during human navigation: gender-different neural networks as substrate of performance. Nat Neurosci 2000; 3(4): 404-8.

[81] Nowak K, Mix E, Gimsa J, Strauss U, Sriperumbudur KK, Benecke R, Gims U. Optimizing a rodent model of Parkinson's disease for exploring the effects and mechanisms of deep brain stimulation. Parkinsons Dis 2011; 2011: 414682.

[82] Wrase J, Klein S, Gruesser SM. Gender differences in the processing of standardized emotional visual stimuli in humans: a functional magnetic resonance imaging study. Neurosci Lett 2003; 348(1): 41-5.

[83] Klein S, Smolka MN, Wrase J.The influence of gender and emotional valence of visual cues on FMRI activation in humans. Pharmacopsychiatry 2003; 36 (Suppl 3): S191-4.

[84] Proverbio AM, Adorni R, Zani A, Trestianu L. Sex differences in the brain response to affective scenes with or without humans. Neuropsychologia 2009; 47(12): 2374-88.

\section{Received: June 24, 2011 \\ (C) Casanova et al.; Licensee Bentham Open.}

This is an open access article licensed under the terms of the Creative Commons Attribution Non-Commercial License (http://creativecommons.org/licenses/by$\mathrm{nc} / 3.0 /$ ), which permits unrestricted, non-commercial use, distribution and reproduction in any medium, provided the work is properly cited. 